\title{
A European perspective of clinical decision-
} making

Nick A Bakalis RN; BSc, PGCert., PGDip., MSc, PhD, Nurse Researcher, School of Nursing, ATEl Patras, Greece

E-mail: nb1972@hotmail.com

Key Words: decision making * England * Greece * nursing education

SUMMARY

- The competence of health care personnel is based on their prior education and the knowledge it provides.

- $\quad$ Nurses need to develop the appropriate knowledge and skills to become effective decision-makers.

- This study found that English final year student nurses had better knowledge of anatomy and physiology of the normal heart.

- The results also showed that coronary care nurses in England made better quality clinical decisions in the acute and recovery phases of myocardial infarction than their Greek counterparts.

- The assumption that better educated nurses made better quality clinical decisions was supported.

\section{INTRODUCTION}

With the introduction of coronary care units (CCUs) in 1962, the mortality rate of patients admitted to hospital with acute myocardial infarction (MI) fell from $30 \%$ in 1975 to $15 \%$ in 1995 (Moser \& Dracup, 1996). Furthermore, the average length of stay for the acute MI patient had decreased from 21 days in 1970, to 6 days in 1995 (Wilson \& Waugh, 1996). The reduction of mortality rate and reduced in-patient stay was significantly and positively affected by nurses who were able to interpret the electrocardiograph (ECG) findings displayed on the cardiac monitors (Adams \& Perez, 1991), recognise the significance of changes in cardiac rate and assume a decision-making role in an emergency situation (Caunt, 1992). This, combined with increased levels of responsibility associated with technology and specialisation, requires nurses to be autonomous decision-makers (Boney \& Baker, 1997).

To explore this concept further the researcher was interested in investigating issues surrounding nurses' decision-making. Based on the researcher's observations and experience of working in more than one European country, an assumption was made that better educated nurses make better clinical decisions.

The aim of this research was first to seek explanations for similarities, second to gain a greater awareness and a deeper understanding of issues such as education and clinical decision-making, and third to adapt (where possible) foreign ideas and practices to the needs of the researcher's own country.

\section{CLINICAL DECISION-MAKING}

Decision-making is regarded as an essential component of the nursing role (Caputo \& Mior, 1998). Models of practice are essentially decision-making models and thus synonymous with accepted definitions of professional activity (Rhodes, 1985). Drummond (1996) describes decision-making as a process that involves steps or actions that one might take in solving problems. Despite the extensive measurement of decision-making, most authors have defined the decision-making process rather than stating what a decision is.

Previous research published in the literature in regards to clinical decisionmaking shows that decision-making strategies have been investigated using both quantitative and qualitative approaches. Those that have taken a quantitative approach have largely examined the outcomes of the decisions, and have used mathematical (statistical) techniques for analysis, whereas those that have taken a qualitative approach have concentrated on exploring how decisions were made and the processes used in decision-making. Additionally, some studies have also examined the factors that influence decision-making (Hamers et al., 1994; Erlen \& Sereika, 1997; Luker et al., 1998).

A variety of different methods has been used to investigate decisionmaking. When designing a study that investigates issues of decisionmaking the aim of the study needs to be considered. If the output of a decision-making process is the aim of the investigation, observation, interviews and surveys are appropriate techniques (Fonteyn et al., 1993). However, when the aim of the investigation is to identify how decisions are made, the above methods are problematic. Fonteyn et al. (1993) stated that interviewing subjects about what they think they do is fraught with problems concerning accuracy and validity, as what people say they do and how they actually behave may be entirely different.

Finally, if the aim of the investigation is the outcome of the decision, then decision analysis can be used. Henry (1995) asserts that decision analysis can assist nurses to analyse and compare alternative actions in a systematic manner. Patient simulations present to the subjects who choose one of the alternative options (Pratt et al., 1995). Patient simulation provides a realistic clinical situation in which subjects make decisions without the influence of the clinical authority and with no risk to a patient (Woodbury, 1984). However, Offredy (1998) points out that the use of decision analysis is questionable when addressing management of symptoms and the importance of patients' action and feedback in the decision-making process.

Decision analysis theory was used as a theoretical base for the second part of this study. Decision analysis theory breaks down the decision into a number of actions and coding the data into numerical values allows analyses to be undertaken on each decision.

Knowledge and clinical experience have been found to be the most important factors influencing clinical decision-making by several researchers (Benner \& Tanner 1987; Saad \& Hammers, 1997; Bucknall \& Thomas, 1997; Caputo \& Mior, 1998). The knowledge a nurse brings to the diagnostic task plays a critical role in determining how the problem will be interpreted and which items of clinical information will be attended to (Jones, 1988). Thus, reference to prior content knowledge is a prelude to decision-making (Corcoran, 1986) and has an important influence on the proficiency of the decision-making that takes place (Moore, 1996).

A number of studies identified the need to base clinical decision-making in evidence-based practice (Alexander, 1997). Clinical guidelines, protocols and care pathways are approaches that facilitate evidence-based 
practice. It is generally accepted that health care personnel work towards providing the best possible outcomes of care and treatment through the implementation of evidence-based practice.

Psychological stress occurs during the decision-making process (Jannis \& Mann, 1982). Critical care areas are described as stressful environments because of the complexity of patient health problems and the increasing use of high technology (Duff et al., 1996). In addition, critical care nurses make many decisions in a short period of time often with little information and a high degree of uncertainty (Hicks, 1999). These decisions may have a profound impact on the patient's life and thus nurses are aware of the serious consequences of mistakes. Also, the mutually supportive nurse-physician relationship in the critical care unit is paramount to the nurse's decision-making (Baggs et al., 1997).

The literature indicates that the role of critical care nurses has a profound effect on clinical decision-making (Bucknall \& Thomas, 1995). Critical care nurses have a multidimensional role to fulfill and this is due to work environment and patient complexity. More precisely, coronary care units (CCUs) are not a quiet and calm environment but one with flashing lights, frequent alarms and highly technological equipment. Nurses must have an advanced knowledge in cardiac nursing, specifically pathophysiology and pharmacology knowledge, be highly skilled in the use of the equipment and develop characteristics such as alertness, sensitivity and a fully understanding of body haemodynamics in order to be able to interpret the patients' needs and take the necessary actions. Nevertheless, nursing care should be directed not only towards physical problems but also towards the patient's psychosocial well being (Jowett \& Thompson, 1988). Nurses also have a teaching, counseling and supporting role to fulfill.

\section{METHODOLOGY}

Aims and objectives of the study

The overall aim of the study was to examine the philosophical assumption that better educated nurses make better quality clinical decisions. The objectives were to:

- determine the level of cardiac knowledge in Greek and English final year student nurses and to check the theoretical credibility (phase 1),

- identify what clinical decisions coronary care nurses make in the acute and recovery phases of $\mathrm{Ml}$ in Greece and England (phase 2).

Phase 1

\section{Sample}

The phase 1 sample included final year student nurses of degree and diploma courses in Greece $(n=87)$ and England $(n=74)$. Four institutions (two in each country) were selected randomly to participate in the study. In order to recruit nurses to participate in this study, letters were sent to the heads of nursing departments in Greece and England, explaining the purpose and nature of the study and asking permission to approach final year student nurses to participate. After permission was given from the Universities Authority, all final year student nurses were included in the study.

\section{Data collection}

Pictographs (testing knowledge in a pictorial form) were used as a method of collecting data. Knowledge of anatomy and physiology was chosen as the subject crossed cultural boundaries and omitted problems of translation. Pictographs used were two anatomical cardiac diagrams and one normal electrocardiograph trace with a total of 20 blank items for labeling data. Thus, the minimum total score was 0 and the maximum was 20 . In addition to diagrams, expert panels in both countries were used to avoid culture bias. Each expert panel consisted of four academics (two from each country). All were nurse teachers with at least seven years' teaching experience and a background of cardiac nursing. In order to evaluate the translation, the Greek panel received two copies (Greek and English) of the pictographs since they studied at English universities. Both panels agreed that the structure and themes were appropriate.

\section{Data Analysis}

Data were entered and analysed using version 10.0 of the Statistical Package for Social Scientists (SPSS Inc.). A significance level of $p<0.05$ was chosen. A graphical method (nomogram) (Altman, 1991) and the sample size tables of Mahin (1997) were used in order to calculate the appropriate sample size for the second study (decision-making).

\section{Results}

Most Greek students (90.9\%) were aged between 21 and 23 with a mean age of 22.5 years, and were female $(74.7 \%)$ whereas most English students $(75.5 \%)$ were aged between 22 and 29 years with a mean age of 27 , and were female (94.6\%).

English student nurses scored significantly higher in their cardiac knowledge than their Greek counterparts $(p<0.005)$ (see Table 1).

\begin{tabular}{|c|c|c|c|c|c|c|}
\hline & $\mathbf{n}$ & $\begin{array}{c}\text { mean } \\
\text { score }\end{array}$ & SD & $\boldsymbol{t}$ & df & Significance \\
\hline Greek & 87 & 11.6425 & 3.208 & \multirow{2}{*}{-7.35} & \multirow{2}{*}{158.5} & $\mathrm{P}<0.005$ \\
\hline English & 74 & 15.1676 & 2.875 & & & \\
\hline
\end{tabular}

Table 1: $t$-test of total score by nationality

Phase 2

\section{Sample}

The hypothesis testing study (phase 1) provided the data for the power analysis for the clinical decision-making study sample size. It was calculated that a sample of 60 nurses (30 in each country) would be needed for the second study with a probability power of $99 \%$. However, following the advice of a statistician it was decided to increase the sample size to 100 (50 in each country). This was done to enable the use of statistical analysis methods such as multiple regression and to increase generalisability. Sixteen hospitals (eight from each country) were selected randomly.

Letters were sent to the nurses in charge of each CCU in Greece and England, explaining the study and requesting permission to approach Registered Nurses (RNs) to participate.

\section{Data collection}

The research was carried out in a private room with chairs and a table available. Disturbances were minimised by using a sign outside the room. The researcher gave a scenario to each participant to read and then presented them with possible choices. The researcher asked the participant to make only one choice that most closely reflected their normal practice. The participant then indicated their choice by touching the card. When they made a decision, they received more information about their choice. For example if they had chosen to check the vital signs, they were given this information orally from the researcher. Then the next row ( 5 choices) was presented and then asked to make another choice, and so on until the scenario ended. The time to complete the instrument varied from 20 to 25 minutes. It was a very smooth process and no problems were encountered.

$\mathrm{Q}$ methodology or Q-sort (clinical decision-making cards) was used to measure quality clinical decisions between Greek and English coronary care nurses. Q-methodology is a means of obtaining data in which subjects sort statements into categories according to their attitudes toward, or rating, of the statements. Eight scenarios (four were based in the acute cardiac area and four focussed on the recovery phase of a cardiac event) were constructed. The scenarios with an acute care focus included issues involving chest pain, bradycardia, tachycardia and basic life support. Anxiety, sleeping difficulties, breathing difficulties and vomiting were issues included in scenarios used for the recovery phase. 
A modified scoring system adopted by Williamson (1965) was used. The scoring system was as follows:

\section{- helpful (+2),}

- facilitate but not essential $(+1)$,

- $\quad$ neither promotes nor impedes care (0),

- unnecessary and/or cause discomfort $(-1)$

- inappropriate (-2).

The minimum score was -4 since two inappropriate answers ended the scenario and the maximum score was varied according to the scenario. Five choices in each row were constructed. The helpful decision-making pathways were found from the literature (European guidelines, nursing books). The researcher, who has experience in coronary care work, constructed the alternative pathways. An expert panel in both countries was used to check the structure, content, alternative pathways and translation of the scenarios. All six nurses (three in each country) in the expert panel had nursing degrees and at least 7 years' clinical experience in CCU. Cards were laminated in A5 sizes. Simulated scenarios were laminated on a larger scale to show difference.

\section{Data analysis}

Data were analysed in two ways. First, the overall score for each scenario was calculated by adding the sum of positive and negative answers for each participant (Williamson, 1965). Second, to measure the quality of clinical decision-making, four categories were developed to tabulate the (overall) score of each individual. Scores were grouped as very poor, poor, good and very good.

\section{Results}

English coronary care nurses were aged between 25 and 50 years with a mean age of 30.5 years while the Greek nurses aged between 24 and 43 years with a mean age of 33.1 years (see Figure 1).

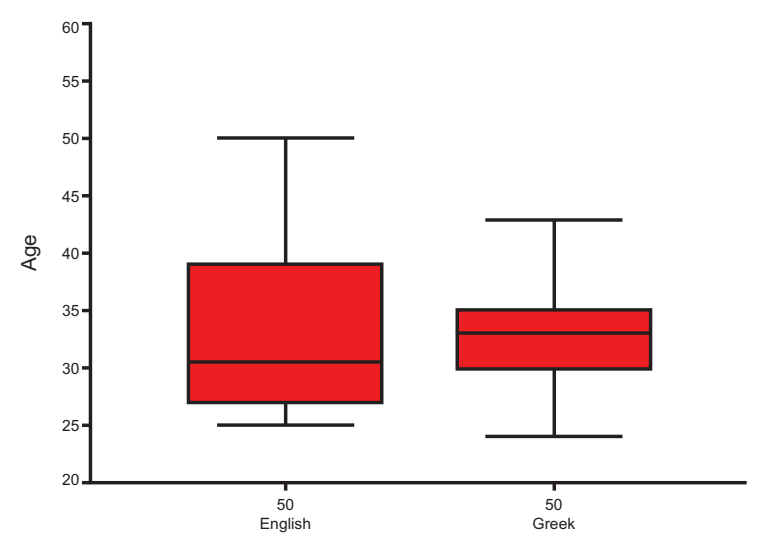

Figure 1: Age distribution of English and Greek coronary care nurses

The vast majority of English nurses (70\%) had worked between 1 and 5 years in CCU whereas $50 \%$ of Greek nurses had worked between 6 and 13 years and $50 \%$ between 1 and 5 years. Furthermore, the majority of English CCU nurses (64\%) had undertaken further academic studies since registration. In contrast, a large number of Greek nurses $(76 \%)$ had not undertaken any academic studies since registration.

Overall, English CCU nurses scored higher in the acute phase (mean 25.06, SD 8.93, range 5-45) and recovery phases of MI (mean 43.66, SD 6.34 , range 25-57) than the Greek nurses in the acute (mean 23.38, SD 8.99 , range 9-42) and recovery phases (mean 37.10, SD 10.61, range1758) $(t=0.937, d f=98, \mathrm{p}>0.05$ for acute phase and $t=3.752, d f=98, \mathrm{p}$ $<0.001$ for recovery phase) (see Figure 2).

In the acute phase, both Greek and English CCU nurses made good clinical decisions in all scenarios. Interestingly, Greek nurses made more very good clinical decisions in scenarios 1 and 3 than the English nurses. In contrast, in the recovery phase English CCU nurses made very good clinical decisions in all scenarios while the Greek nurses made good decisions in scenarios 6, 7 and 8 and very good clinical decisions in scenario 5 (see Table 2).
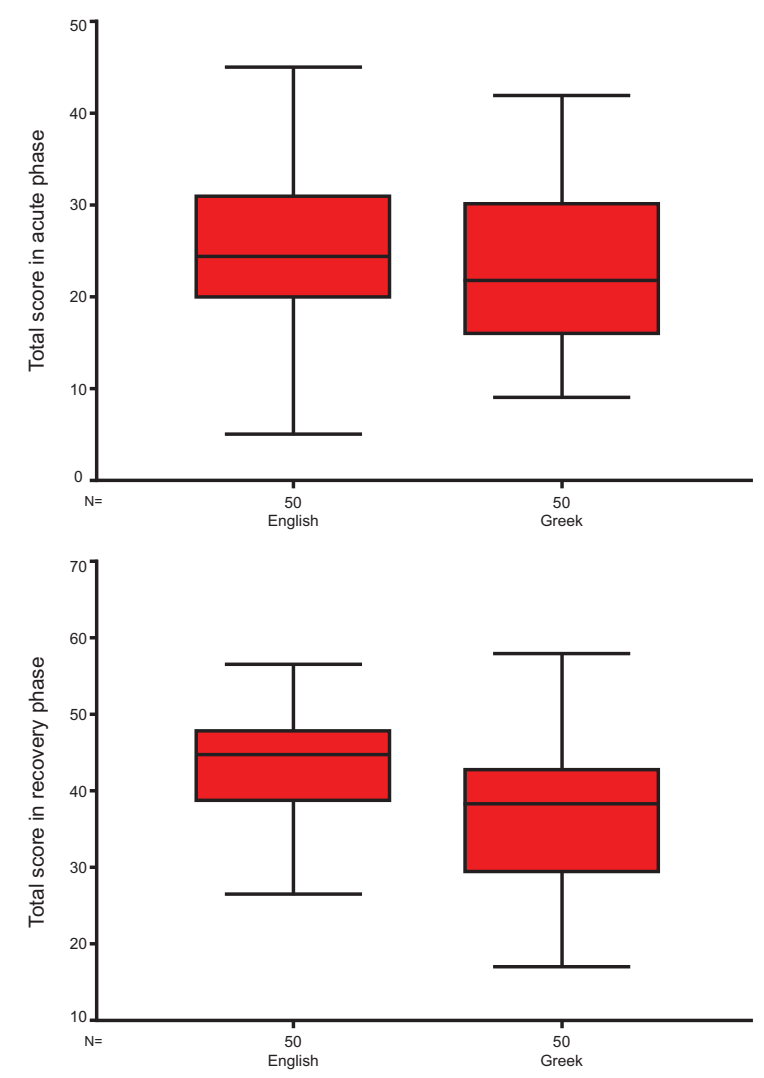

Figure 2: Total score of decision-making in the acute and recovery phases of MI

\begin{tabular}{|c|c|c|c|c|c|c|c|c|}
\hline & \multicolumn{4}{|c|}{ Greece } & \multicolumn{4}{c|}{ England } \\
\hline Rating & $\begin{array}{l}\text { Very } \\
\text { Poor }\end{array}$ & Poor & Good & $\begin{array}{c}\text { Very } \\
\text { Good }\end{array}$ & $\begin{array}{c}\text { Very } \\
\text { Poor }\end{array}$ & Poor & Good & $\begin{array}{c}\text { Very } \\
\text { Good }\end{array}$ \\
\hline Scenario 1 (n) & 1 & 11 & 27 & 11 & - & 11 & 35 & 4 \\
\hline Scenario 2 (n) & 1 & 19 & 22 & 8 & 1 & 19 & 19 & 11 \\
\hline Scenario 3 (n) & 1 & 12 & 26 & 11 & 2 & 10 & 30 & 8 \\
\hline Scenario 4 (n) & - & 10 & 22 & 18 & - & 10 & 18 & 22 \\
\hline Scenario 5 (n) & - & 6 & 9 & 35 & - & - & 2 & 48 \\
\hline Scenario 6 (n) & - & 5 & 24 & 21 & - & 2 & 12 & 36 \\
\hline Scenario 7 (n) & - & 8 & 23 & 19 & - & 2 & 17 & 31 \\
\hline Scenario 8 (n) & - & 10 & 23 & 17 & - & 1 & 18 & 31 \\
\hline
\end{tabular}

Table 2: Quality of clinical decision-making in Greece and England

The data show that English nurses made significantly better quality clinical decisions in the recovery phase of MI than their Greek counterparts $(p<$ $0.005)$. In the acute phase, in scenarios one and three, Greek nurses made better quality clinical decisions $(p>0.05)$ and in scenarios two and four, English nurses made better quality clinical decisions $(p>0.05)$. Scenario 1 (chest pain) is shown over page.

The data also indicate that that age $(r=0.24, d f=100, p<0.05)$, years working in CCU $(r=0.33, d f=100, p<0.01)$, academic studies $(r=0.25$, $d f=100, p<0.05)$ and medical cover $(r=0.26, d f=100, p<0.01)$ were positively correlated with the total score in two phases. 


\section{Scenario 1}

A patient has just been admitted to your unit with myocardial infarction. The patient is a 65 year old, male, with no previous medical history of cardiac problems. He has been seen by a doctor. Suddenly, the patient complains of acute chest pain. Each card gives you a choice of possible action. Choose one card which reflects your normal practice.

\section{DISCUSSION}

Decision-making in nursing is concerned with managing a range of information from diverse sources in order to make a professional clinical judgment (Gambrill, 1990). Decision-making is important at all levels of the managerial process and at different levels of complexity. Although the literature indicates that most studies examine how decisions are made and the numerous factors that influence those decisions, there is a paucity of research regarding what actual clinical decisions nurses make in clinical practice and the quality of these decisions.

In the acute phase of $\mathrm{Ml}$, coronary care nurses are confronted with patients whose health status changes rapidly and thus interventions are required to prevent deterioration. Because nurses are held responsible and accountable for clinical decisions, the nurse's role has expanded and become more specialised. The recovery phase for patients after a cardiac event, on the other hand, is characterised by the fact that patient status is stable and the rehabilitation phase is necessary.

The findings from this study revealed that English coronary care nurses made better clinical decisions in all scenarios of the recovery phase of Ml. However, in scenarios 1 (chest pain) and 3 (tachycardia) the results showed that Greek coronary care nurses made better quality (although not statistically significant) clinical decisions than their English counterparts. This may be due to the fact that medical support in CCUs in Greece is not always available on a 24-hour basis. Therefore, under emergency situations, when doctors are absent, nurses independently make decisions and implement actions that normally would require doctor involvement. These findings are supported by Prescott et al. (1987) who revealed that in life-threatening situations critical care nurses made independent decisions and implemented actions and then let the physician know about the situation.

According to Hooft (1990) professional autonomy involves the nurse's freedom to act in the best interest of the patient. Autonomy is created by society and by its institutions, in this case the hospital. Hooft (1990) adds that professional autonomy is a social phenomenon and thus external to the nurse. It may be that social perception of nursing is different in Greece and England.

The study also revealed the importance of clinical experience in clinical decision-making. The more experience coronary care nurses had, the more in-depth clinical decisions they made. Benner and Tanner (1987) support this finding.

Another factor, which was found to influence clinical decision-making, was pre-registration education. The hypothesis testing study (phase 1) found that English students nurses had greater cardiac knowledge (mean score 15.1). The more highly educated coronary care nurses are the better is the quality of their decisions, supporting the philosophical assumption. Nurses in Greece and England have similar secondary education and entry requirements. Consequently, the wide variation in scores between Greek and English students nurses may be due to differences in the nursing curriculum and more specifically in nursing content, teaching methods and teachers' qualifications.

The use of eight scenarios to examine decision-making in the acute and recovery phases of $\mathrm{Ml}$ were authentic, it gave the opportunity to identify clinical decisions coronary care nurses made in practice. The literature and the results of this study, indicate that the two phases are different in terms of management and philosophy of patient care.

\section{LIMITATIONS}

The main limitation of this study relates to the issue of cultural bias. For the present study, pictographs, expert panels and literature search were used in order to minimise bias and produce valid and reliable instruments. The study is also limited by the relatively small sample size.

\section{RECOMMENDATIONS}

While much useful information has been gained from this study, experience of using the instruments with a larger group of respondents is desirable. The study examined the quality clinical decisions in the acute and recovery phases of $\mathrm{MI}$ in Greece and England however, the extent to which the quality of these decisions impact on the quality of patient care and patient outcome, is something that needs further exploration in future research.

\section{CONCLUSIONS}

In day-to-day nursing practice, nurses are constantly making decisions. One of the major roles of professionals is to make, or to participate in, making decisions in conditions of uncertainty, especially in situations that have major life consequences, like areas of coronary care units.

This study revealed that coronary care nurses made better clinical decisions in the recovery phase of MI than the acute phase. This reflects the fact that the acute phase involves more medical intervention and the recovery phase more nursing intervention. This indicates that the nursing role might strengthen at the rehabilitation and adaptation stage of MI. Moreover, English coronary care nurses made better quality clinical decisions in the acute and recovery phases of MI. Pre-registration education may have an impact in decision-making.

In the $21^{\text {st }}$ century, nurses will need to make independent and confident clinical decisions. Therefore, nursing clinical practice should be secured by providing opportunities for nurses to keep up-to-date with technological and pharmacological changes in order to practise professionally and meet the challenging needs of health care.

\section{REFERENCES}

Adams DL, Perez SR (1991) Caring for the acute myocardial infarction patient: a nursing perspective. Henry Ford Hospital Medical Journal 39 (3 \& 4), 251-255.

Alexander C (1997) Influencing decision making. Nursing Standard 11 (38), 39-42.

Altman D (1991) Practical statistics for medical research. Chapman \& Hall, London.

Baggs JG, Schmitt MH, Mushlin Al, Eldredge DH, Oakes D, Hutson AD (1997) Nurse-physician collaboration and satisfaction with the decision making process in three critical care units. American Journal of Critical Care 6 (5), 393-399.

Benner P, Tanner C (1987) How expert nurses use intuition. American Journal of Nursing 87, 23-31.

Boney J, Baker JD (1997) Strategies for teaching clinical decisionmaking. Nurse Education Today 17, 16-21.

Bucknall T, Thomas S (1997) Clinical decision making in critical care Australian. Journal of Advanced Nursing 13 (2), 10-17.

Caputo LA, Mior SA (1998) The role of clinical experience and knowledge in clinical decision making. Topics in Clinical Chiropractic 5 (2), 10-18.

Caunt JE (1992) The changing role of coronary care nurses. Intensive \& Critical Care Nursing 8, 82-93.

Corcoran SA (1986) Task complexity and nursing expertise as factors in decision making. Nursing Research 35 (2), 107-112.

Duff LA, Kitson AL, Seers K, Humphris D (1996) Clinical guidelines: an introduction to their development and implementation. Journal of Advanced Nursing 23, 887-895. 
Drummond H (1996) Effective Decision Making, $2^{\text {nd }}$ Ed. Kogan Page, London.

Erlen J, Sereika S (1997) Critical care nurses' ethical decision-making and stress. Journal of Advanced Nursing 26 (5), 953-961.

Fonteyn ME, Kuipers B, Grobe S (1993) A description of think aloud method and protocol analysis. Quarterly Health Research 3 (4), 430-441.

Gambrill E (1990) Critical Thinking in Clinical Practice. Jossey-Bass, Oxford.

Hamers JP, Saad H, Schumacher JN (1994) Factors influencing nurses' pain assessment and interventions in children. Journal of Advanced Nursing 20, 853-860.

Henry SB (1995) Nursing informatics: state of the science. Journal of Advanced Nursing 22 (6), 1182-1192.

Hicks F (1999) Developing and supporting expansion of the nurse's role. Nursing Standard 11 (24), 41-45.

Hooft S (1990) Moral education for nursing decisions. Journal of Advanced Nursing, 15: 210-215.

Jannis L, Mann L (1982) Stress, attitudes and decisions. Praeger Publications, New York.

Jones JA (1988) Clinical reasoning in nursing. Journal of Advanced Nursing 13, 185-192.

Jowett NI, Thompson DR (1988) Comprehensive Coronary Care. Scutari Press, Middlesex.

Luker K, Hogg C, Austin L, Ferguson B, Smith K (1998) Decision making: the content of nurse prescribing. Journal of Advanced Nursing 27, 657-665.
Mahin D (1997) Sample size tables for clinical studies. Blackwell Science, London.

Moore PA (1996) Decision Making in Professional Practice. British Journal of Nursing 5 (10), 635-640.

Moser D, Dracup K (1996) Is anxiety early after myocardial infarction associated with subsequent ischemic and arrhythmic events? Psychosomatic Medicine 58, 395-401.

Offredy M (1998) The application of decision making concepts by nurse practitioners in general practice. Journal of Advanced Nursing 28 (5), 988-1000.

Pratt JW, Raiffa H, Schlaifer R (1995) Introduction to Statistical Decision Theory. MIT Press, Cambridge.

Prescott P, Dennis K, Jacox A (1987) Clinical decision making of staff nurses. IMAGE: Journal of Nursing Scholarship 19 (2), 56-62.

Rhodes BA (1985) Accountability in nursing: alternative perspectives. Nursing Times, 79(36): 65-66.

Saad H, Hamers J (1997) Decision making and paediatric pain: a review. Journal of Advanced Nursing 26, 946-952.

Williamson JW (1965) Assessing clinical judgement. Journal of Medical Education 40, 180-187.

Wilson R, Waugh S (1996) Cardiac Nursing. W.B.Saunders, Philadelphia

Woodbury PA (1984) Computer assisted evaluation of problem solving skills of primary health care providers. The Journal of Continuing Education in Nursing 15 (5), 174-177. 